PHYSICAL REVIEW D 90, 023008 (2014)

\title{
Entropic-force dark energy reconsidered
}

\author{
Spyros Basilakos ${ }^{1}$ and Joan Solà ${ }^{2,3}$ \\ ${ }^{1}$ Academy of Athens, Research Center for Astronomy and Applied Mathematics, Soranou Efesiou 4, \\ 11527 Athens, Greece \\ ${ }^{2}$ High Energy Physics Group, Department ECM, Universidad de Barcelona, Avenida Diagonal 647, \\ E-08028 Barcelona, Catalonia, Spain \\ ${ }^{3}$ Institut de Ciències del Cosmos (ICC), Universidad de Barcelona, E-08028 Barcelona, Catalonia, Spain
}

(Received 28 February 2014; published 9 July 2014)

\begin{abstract}
We reconsider the entropic-force model in which both kinds of Hubble terms, $\dot{H}$ and $H^{2}$, appear in the effective dark energy (DE) density affecting the evolution of the main cosmological functions, namely, the scale factor, deceleration parameter, matter density, and growth of linear matter perturbations. However, we find that the entropic-force model is not viable at the background and perturbation levels due to the fact that the entropic formulation does not add a constant term in the Friedmann equations. On the other hand, if on mere phenomenological grounds we replace the $\dot{H}$ dependence of the effective DE density with a linear term $H$ without including a constant additive term, we find that the transition from deceleration to acceleration becomes possible, but the recent structure formation data strongly disfavor this cosmological scenario. Finally, we briefly compare the entropic-force models with some related DE models (based on dynamical vacuum energy) which overcome these difficulties and are compatible with the present observations.
\end{abstract}

DOI: 10.1103/PhysRevD.90.023008

PACS numbers: 95.36.+x, 04.62.+v, 11.10.Hi

\section{INTRODUCTION}

The discovery of the cosmic acceleration (see [1-8] and references therein) has opened a new window in trying to understand the Universe. Despite the mounting observational evidence on the existence of the accelerated expansion of the Universe, its nature and fundamental origin is still an open question, challenging the very foundations of theoretical physics. While the simplest possibility is a rigid cosmological constant (CC) term $\Lambda$ for the entire history of the Universe, with the advent of quantum theory and quantum field theory (QFT), the $\Lambda$ term has been associated with the vacuum energy density, $\rho_{\Lambda}=\Lambda /(8 \pi G)$, and this association is at the root of the acute cosmological constant problem [9], perhaps one of the most pressing conundrums of fundamental physics ever-see, e.g., [10] for a recent review.

The difficulties inherent to the CC problem suggest that we adopt a more dynamical perspective. This has led to various forms of dynamical dark energy (DE) as a possible substitute for the rigid CC term. Along these lines one may consider a general mechanism that is responsible for cosmic acceleration which is based either on a modified theory of gravity or on the existence of some sort of DE which is related to the existence of new fields in nature.

Perhaps the most popular idea in the past was to find a scalar field capable of dynamically adjusting the vacuum energy to zero (or, in fact, to the tiny number $\rho_{\Lambda 0} \sim$ $10^{-47} \mathrm{GeV}^{4}$ that has been measured in recent times), with the hope of solving the old CC problem [9]. The first attempts in this direction are quite old [11-13]. It is only in more recent times that this approach took the current popular form of quintessence [14,15] and was mainly applied to explain the possible dynamical character of the DE, with an eye at solving the "cosmic coincidence" problem. The DE ideas since then have branched off into multiple different formulations; see, e.g., [16-33] and references therein. In particular, we have also the dynamical models of the vacuum energy based on the renormalization group approach [34-38]. As we will see, these models bare a close (but distinctive) relation with the entropic-force models.

Amongst the variety of DE models, the so-called entropic-force dark energy has recently gained a lot of attention in cosmology. The notion of entropic force was suggested by Verlinde [39], who proposed that the gravitational field equations can be derived from the second law of thermodynamics in a way that would render the gravity force quite literally as a kind of "entropic force," i.e., a force related to the change of entropy. Its implementation in cosmology $[40,41]$ is based on the assumption that the horizon can play the role of a holographic screen ${ }^{1}$ (see also [45]).

Such a screen would induce a force $\mathbf{F}=T \nabla S$ on a nearby test particle, where $T$ is the temperature of the

\footnotetext{
${ }^{1}$ Let us mention that in Jacobson's [42] and Padmanabhan's approaches to entropic gravity [43], the formulation is much more general and is not affected at all by our analysis here, which is restricted to the specific entropic-force version [39] and its cosmological implementation in [40,41]. See also Ref. [44] for the difficulties of interpreting entropic forces in Newtonian gravity.
} 
screen and $\nabla S$ is the change of entropy associated with the information contained in it (which involves a large number of d.o.f.). The change of entropy when the radius of the Hubble horizon, $R_{H}=1 / H$, increases by $d r$ is simply $d S_{H}=2 \pi\left(R_{H} k_{B} M_{P}^{2}\right) d r$, where $M_{P}$ is the Planck mass and $k_{B}$ is Boltmann's constant. From the pressure exerted by the entropic force on the cosmological expansion, $P=F /$ $A=-(T / A) d S_{H} / d r$, and estimating the horizon temperature by the de Sitter temperature, $T=\left(\hbar / k_{B}\right)(H / 2 \pi)$, one finally obtains $P=-M_{P}^{2} H^{2} / 4 \pi$. The minus sign in the pressure is of course the characteristic feature of the accelerated expansion in this entropic version. This framework, therefore, suggests that the entropic force leads to an effective DE density $\rho_{\mathrm{DE}}$ which is dynamical and evolves as the square of the Hubble rate: $\rho_{\mathrm{DE}} \propto H^{2}$.

In this formulation, the DE does not exist as an exotic energy component of the Universe, but as an effective force acting outwards to the cosmic horizon, thereby accelerating the evolution of the Universe. This particular scenario is the so-called "entropic-force cosmology," and it was first proposed in Refs. [40,41] and later on discussed by various authors-see, e.g., [44,46-50].

One crucial question is what classes of entropic-force models are allowed in cosmological studies. Specifically, we are interested in testing the validity of the entropic-force DE both at the background and cosmic perturbation levels. In order to do so, we need to define the Hubble parameter and the growth of matter perturbations in the linear regime as a function of redshift and then to check whether the above functions could lead to reliable cosmological results.

The layout of the article is the following. In Sec. II, we present the main ingredients of the dynamical problem under study. In Secs. III and IV, we provide the analytical solutions for the Hubble and growth factor, respectively. Finally, we draw our conclusions in Sec. V.

\section{ENTROPIC-FORCE MODELS AND EFFECTIVE DARK ENERGY}

The nature of the entropic-force models [39] is essentially connected with a surface effect from the horizon. From the latter one may utilize the gravitational action for space-times with boundaries [51]. This is achieved by adding the boundary action term $S_{B}$ to the standard Einstein-Hilbert action, $S_{\mathrm{EH}}$, namely,

$$
S_{\mathrm{EH}}+S_{B}=\frac{1}{16 \pi G} \int_{\mathcal{M}} d^{4} x \sqrt{|g|} R+\frac{1}{8 \pi G} \int_{\partial \mathcal{M}} d^{3} y \sqrt{|h|} K .
$$

Here $R$ is the Ricci scalar, $h$ is the determinant of the metric $h_{a b}$ on the boundary $\partial \mathcal{M}$, induced by the bulk metric $g_{\mu \nu}$ of $\mathcal{M}$, and $y^{a}$ are the coordinates on $\partial \mathcal{M}$. Furthermore, $K$ is the trace of the second fundamental form (or extrinsic curvature); if $n^{\mu}$ is the normal on the boundary, it can be written as $K=\nabla_{\mu} n^{\mu}$. The overall action is
$S=S_{\mathrm{EH}}+S_{B}+S_{m}$, where $S_{m}$ represents the ordinary matter contribution. From the technical point of view, the precise definition of the boundary term $S_{B}$ should actually include an overall sign, which is plus or minus depending on whether the hypersurface $\partial \mathcal{M}$ is spacelike $\left(n^{\mu} n_{\mu}=+1\right)$ or timelike $\left(n^{\mu} n_{\mu}=-1\right)$, respectively. We exclude null surfaces for this consideration.

The precise coefficient in front of the boundary integral $S_{B}$ is chosen in such a way that the surface terms generated from the metric variation of $S_{\mathrm{EH}}$ are exactly canceled by the metric variation of $S_{B}$, provided the variation $\delta g^{\mu \nu}$ is performed in such a way that it vanishes on $\partial \mathcal{M}$, i.e., provided the induced metric $h_{a b}$ on the boundary is held fixed. It follows that, in the presence of $S_{B}$, the standard form of Einstein's equations is preserved even if the space-time has boundaries.

As the surface terms emerging from the variation of $S_{\mathrm{EH}}$ are canceled by $\delta S_{B}$, one may assume that if the total action would not contain $S_{B}$, the contribution of the aforementioned surface terms to the field equations would be of the order of the effect induced by $S_{B}$, estimated as $R$ times the prefactor $1 /(8 \pi G)$ in $S_{B}$. In the Friedmann-LemaitreRobertson-Walker metric with flat space slices, this reads $\left(12 H^{2}+6 \dot{H}\right) /(8 \pi G)$, with $H=\dot{a} / a$ and $\dot{H}=d H / d t$. This is presumably the kind of consideration made by Easson et al. [40]. They also argued that since this is probably just a rough estimate of the effect, one should generalize the corresponding acceleration equation for the scale factor in the form

$$
\frac{\ddot{a}}{a}=-\frac{4 \pi G}{3}\left(1+3 \omega_{m}\right) \rho_{m}+C_{\dot{H}} \dot{H}+C_{H} H^{2},
$$

where $\omega_{m}=p_{m} / \rho_{m}$ is the equation of state $(\mathrm{EoS})$ for the matter fluid (with $\omega_{m}=0$ and $1 / 3$ for nonrelativistic and relativistic matter, respectively). The second cosmological equation is the generalized Friedmann's equation:

$$
H^{2} \equiv\left(\frac{\dot{a}}{a}\right)=\frac{8 \pi G}{3}\left[\rho_{m}(t)+\rho_{\mathrm{DE}}(t)\right]
$$

where we always assume flat space metric. In the above expression, we have defined

$$
\rho_{\mathrm{DE}}(t)=\frac{3}{8 \pi G}\left[C_{\dot{H}} \dot{H}(t)+C_{H} H^{2}(t)\right] .
$$

It plays the role of effective DE for the generalized cosmological model. Despite the two equations above that imply $\omega_{\mathrm{DE}}=P_{\mathrm{DE}} / \rho_{\mathrm{DE}} \equiv-1$, the entropic-force model is not a time varying vacuum model since it does not have a $\Lambda \mathrm{CDM}$ limit; that is to say, we cannot obtain a constant $\rho_{\mathrm{DE}}$ behavior at any time in its Hubble expansion history.

Let us emphasize at this point an issue previously mentioned in passing, but one that is important when we consider the status of the basic Eqs. (2) and (4). These 
equations are not necessarily derived from first principles, such as a fundamental action. As a matter of fact, in the most general entropic-holographic formulations [42,43], one considers that the gravitational field equations are not necessarily inferred from a fundamental action at the present macroscopic level of description. The field equations can nevertheless provide, in principle, a fully satisfactory account of all the basic phenomena known to date. In such general "emergent gravity formulation," the ultimate origin of gravity is claimed to lie in some fundamental degrees of freedom quite different from the metric variables, namely, degrees of freedom which are completely unknown to us at present. In this sense, the field equations under discussion are also thought of as just effective field equations without having a known fundamental action behind. However, we should also remark that these particular equations pertain to a restricted formulation of the general entropic approach called entropic-force gravity, and it is our aim to discuss the phenomenological status of this restricted version of the entropic approach concerning the cosmological implications first suggested in Ref. [40]. In this sense, our conclusions concerning the phenomenological viability of these models cannot be extended beyond the domain of this formulation.

Implicit herein is the fact that matter is not conserved in the entropic-force models. Indeed, Eqs. (2) and (3) can be used to show that matter and the effective DE (4) exchange energy through the generalized conservation law:

$$
\dot{\rho}_{\mathrm{DE}}+\dot{\rho}_{m}+3\left(1+\omega_{m}\right) H \rho_{m}=0 .
$$

This equation is, therefore, not an independent one, but it is useful to show explicitly that there is a continuous exchange of energy between matter and the generalized DE given by Eq. (4). Such an equation is actually enforced by the Bianchi identity, which implies $\nabla^{\mu} \tilde{T}_{\mu \nu}=0$ for the total energy-momentum tensor $\tilde{T}_{\mu \nu}$, obtained from the ordinary matter contribution, $T_{\mu \nu}$, plus the variable DE density (4). This is in contradistinction to what was assumed in [40]. Let us note that in [41] the treatment of the covariant matter conservation law was different from the previous one by the same authors in [40], and the modified law was presented in the form

$$
\dot{\rho}_{m}+3\left(1-C_{H}\right)\left(1+\omega_{m}\right) H \rho_{m}=0 .
$$

However, this equation is only approximate. It can be easily derived, e.g., from our Eqs. (3) and (4) after neglecting the $C_{\dot{H}}$ contribution. Below we will present the exact solution for $\rho_{m}$ consistent with the full conservation Eq. (5), and then (6) will ensue only as a particular case.

It is not difficult to show that Eqs. (2)-(4) can be combined to produce the following differential equation for the Hubble rate:

$$
\dot{H}+\frac{3}{2}\left(1+\omega_{m}\right) \frac{1-C_{H}}{1-\alpha} H^{2}=0,
$$

where we have defined $\alpha=\frac{3}{2}\left(1+\omega_{m}\right) C_{\dot{H}}$. Using the transformation from the cosmic time $t$ to the scale factor $a$ through $d / d t=a H(a) d / d a$ in Eq. (7) and defining $E(a)=H(a) / H_{0}$, we can easily present the solution of Eq. (7) as follows:

$$
E(z)=(1+z)^{3 \xi_{\omega} / 2},
$$

where $z=a(z)^{-1}-1$ is the redshift, and we have defined the important parameters

$$
\begin{aligned}
\xi_{\omega} & =\xi\left(1+\omega_{m}\right) \\
\xi & =\frac{1-C_{H}}{1-\alpha} .
\end{aligned}
$$

If we consider $C_{H}=3 C_{\dot{H}} / 2$ and $\omega_{m}=0$, then $\xi_{\omega}=\xi=1$ and the above solution boils down to the Einstein-de Sitter model.

The matter density in the entropic-force model is evaluated from

$$
\begin{aligned}
\rho_{m}(z) & =-\frac{1}{8 \pi G\left(1+\omega_{m}\right)} \frac{d H^{2}(a)}{d \ln a} \\
& =\frac{1+z}{3\left(1+\omega_{m}\right)} \rho_{c 0} \frac{d E^{2}(z)}{d z} .
\end{aligned}
$$

Computing this expression and subsequently using it for solving Eq. (5), we can obtain the DE density as well. The final results are

$$
\begin{gathered}
\rho_{m}(z)=\rho_{c 0} \frac{1-C_{H}}{1-\alpha}(1+z)^{3 \xi_{\omega}} \equiv \rho_{m 0}(1+z)^{3 \xi_{\omega}} \\
\rho_{\mathrm{DE}}(z)=\rho_{c 0} \frac{C_{H}-\alpha}{1-\alpha}(1+z)^{3 \xi_{\omega}} \equiv \rho_{\mathrm{DE}, 0}(1+z)^{3 \xi_{\omega}} .
\end{gathered}
$$

In these expressions we identify in the matter era $\left(\omega_{m}=0, \xi_{\omega}=\xi\right)$

$$
\begin{gathered}
\Omega_{m 0}=\frac{\rho_{m 0}}{\rho_{c 0}}=\frac{1-C_{H}}{1-\alpha}=\xi, \\
\Omega_{\mathrm{DE}, 0}=\frac{\rho_{\mathrm{DE}, 0}}{\rho_{c 0}}=\frac{C_{H}-\alpha}{1-\alpha}=1-\xi,
\end{gathered}
$$

which clearly satisfy $\Omega_{m 0}+\Omega_{\mathrm{DE}, 0}=1$, as expected. Furthermore, if one neglects $C_{\dot{H}}$ (hence, $\alpha \simeq 0$ ) we have $3 \xi_{\omega} \simeq 3\left(1-C_{H}\right)\left(1+\omega_{m}\right)$, and then we recover-as a particular case - the solution of the approximate Eq. (6) pointed out by the authors of Ref. [41]. Finally, using Eq. (8) the deceleration parameter $q=-\ddot{a} / a H^{2}$ can be equivalently computed from 


$$
q=-1-\frac{d \ln H}{d \ln a}=-1+\frac{1+z}{E(z)} \frac{d E(z)}{d z}=-1+\frac{3 \xi_{\omega}}{2} .
$$

It is evident that this cosmological model has no inflection point in its cosmic history, i.e., a point where deceleration can change into acceleration. This is because Eq. (15) is redshift independent and, hence, the deceleration parameter maintains sign throughout the cosmic history. As a result the Universe always accelerates or always decelerates depending on the value of $\xi_{\omega}$. In the matter dominated era, namely, $\omega_{m}=0$ and $\xi_{\omega}=\xi$, the accelerated expansion of the Universe $(q<0)$ is obtained for $\xi<\frac{2}{3}$. But in such a case we would also be admitting that the Universe has been accelerating forever, which is of course difficult to accept. Clearly, this feature is the main drawback of this model.

Finally, we would like to mention once more that in this section we have discussed the background evolution via Eqs. (2) and (3) of the DE models considered by the author of the entropic-force models [40]. Although one could define the DE in different ways, our aim was to show that the nominal approach employed by the original authors, the entropic-force model in which both kinds of Hubble terms, $\dot{H}$ and $H^{2}$, appear in the effective DE, is in trouble. In this context, the DE pressure is assumed to satisfy the vacuum equation of state, namely, $P_{\mathrm{DE}}=-\rho_{\mathrm{DE}}$. This is important since in the original model of [40], the above equation of state was not mentioned. In our case, we make this assumption, which is the minimum one we can do. In fact, one can show that if another equation of state would be used the kinds of problems that the entropic model has would remain. For example, if the EoS parameter for the DE would take some arbitrary constant value $\omega_{\mathrm{DE}}$ different from minus one, this would just change the coefficient of $H^{2}$ in Eq. (7), but the solution (8) would take the same form (with a slightly different expression for $\xi_{\omega}$ ) and, hence, the deceleration parameter in Eq. (15) would still be independent of the redshift. As a result, the absence of the inflexion point between deceleration and acceleration would persist.

\section{LINEAR MATTER PERTURBATIONS}

Although finding the background cosmological solution is of course important for our study, no less important is to investigate the structure formation properties, as they play an essential role in the cosmic history. If the entropic-force model discussed in the previous section would be, e.g., the late-time effective behavior of a more complete and fundamental model, we could still ask ourselves quite reasonably if the late-time behavior of that model is compatible with the structure formation data (which is collected precisely in that relevant period).

In this section we provide a detailed answer to such question. To this end we discuss the perturbations in the presence of an entropic-force DE. It will suffice to consider perturbations only for matter, but we have to incorporate the dynamical character of the effective DE in the matter perturbation equations. In other words, the entropic-force $\mathrm{DE}$ is considered variable, but homogeneous in first approximation.

The general study of the linear perturbation equations for a multicomponent fluid has been addressed, e.g., in [52], and we will use the formulation in that paper here-see Eqs. (17), (25), and (27) of that reference (see also $[37,53,54])$. Let us apply those perturbation equations for a system composed of the effective (entropic-force) DE fluid and the matter fluid $\rho_{m}=\rho_{m}(t)$, assuming that there are matter perturbations $\delta \rho_{m}$ but no perturbation in $\rho_{\mathrm{DE}}$. Let us provisionally note here that the inclusion of perturbations of the DE component in these kinds of scenarios, with and without matter conservation, is also possible $[38,55,56]$, but it is not necessary for the present study. We shall further comment below on the viability of this approach.

In this section (and for the entire discussion on perturbations), we just set $\omega_{m}=0$, corresponding to nonrelativistic matter in the epoch of structure formation. The relevant system of first order differential equations reads

$$
\begin{aligned}
\dot{\hat{h}}+2 H \hat{h} & =8 \pi G \delta \rho_{m} \\
\delta \dot{\rho}_{m}+\rho_{m}\left(\theta_{m}-\frac{\hat{h}}{2}\right)+3 H \delta \rho_{m} & =0 \\
\rho_{m} \dot{\theta}_{m}+\left(\dot{\rho}_{m}+5 H \rho_{m}\right) \theta_{m} & =0,
\end{aligned}
$$

where $\hat{h}$ is minus the time derivative of the trace of the metric perturbation $\delta g_{\mu \nu}$, and $\theta_{m}$ the divergence of the perturbed matter velocity [52]. The last equation of the system (16) can be readily rewritten as

$$
\dot{\theta}_{m}+(2 H+Q) \theta_{m}=0,
$$

where we have used Eq. (5) for $\omega_{m}=0$ and defined

$$
Q \equiv \frac{\dot{\rho}_{m}+3 H \rho_{m}}{\rho_{m}}=-\frac{\dot{\rho}_{\mathrm{DE}}}{\rho_{m}} .
$$

Introducing the density contrast $D \equiv \delta \rho_{m} / \rho_{m}$, it is not difficult to show that the first two equations of the system (16) combined with (17) provide the following second order differential equation for $D$ :

$\ddot{D}+(2 H+Q) \dot{D}-\left(4 \pi G \rho_{m}-2 H Q-\dot{Q}\right) D=Q \theta_{m}$.

This equation constitutes a generalization of the basic equation for the matter perturbations in the presence of a dynamical DE density. In the particular case when this term is strictly constant, we have $Q=0$ and the above equation shrinks to the standard one [57] 


$$
\ddot{D}+2 H \dot{D}-4 \pi G \rho_{m} D=0,
$$

which is valid both for CDM and $\Lambda$ CDM cosmologies with the corresponding Hubble function. However, when the DE is time evolving, even if it is perfectly smooth, the correct equation is no longer (20) but (19), a fact which is somehow missed in some approaches in the literature.

The homogeneous version of (19) (i.e., with its rhs equated to zero) was first obtained using a Newtonian approach [53]. Herein we have generalized it within the relativistic formulation in order to discuss if the homogeneous version can also be applied for the models under consideration. First of all, we note that if $|Q|<2 H$ for all $t$, then

$$
\varphi(t) \equiv \int(2 H+Q) d t>0
$$

in any integration interval. Thus, if $\varphi(t)$ increases with time, the solution of (17) is a decaying one:

$$
\theta_{m}(t) \propto e^{-\varphi(t)} \rightarrow 0
$$

In all these cases the product function $Q \theta_{m}$ on the rhs of (19) can be safely neglected. For the class of DE models of the form (4) we can estimate, from the definition (18) of $Q$, that

$$
\frac{|Q|}{H} \sim \frac{\left|C_{\dot{H}} \ddot{H}+C_{H} H \dot{H}\right|}{H^{3}}=\left|\mathcal{O}\left(C_{\dot{H}}\right)+\mathcal{O}\left(C_{H}\right)\right| .
$$

To substantiate the last step, let us differentiate the identity $\dot{H}+H^{2}=-q H^{2}$. Using $q=-\ddot{a} / a H^{2}$ and taking into account that $q \simeq$ const in each epoch, it is easy to see that $\ddot{H} \simeq 2(q+1)^{2} H^{3}$. Together with $H \dot{H}=-(q+1) H^{3}$, these relations provide immediately the estimate (23). It basically tells us that $|Q| / H \ll 1$. Of course this relation holds good insofar as $C_{H}$ and $C_{\dot{H}}$ are expected to be small dimensionless coefficients in the entropic-force scenario. Therefore, Eq. (22) is warranted and we conclude that for the entire class of DE cosmologies (4) we can virtually ignore the rhs of the differential Eq. (19). Hereafter we set it to zero.

For convenience we rewrite the homogeneous form of (19) in terms of the scale factor as an independent variable, which we will use shortly. After straightforward algebra, we arrive at the following expression:

$$
\begin{aligned}
D_{a a}(a)+\left[\frac{3}{a}+\frac{H_{a}(a)}{H(a)}+\frac{Q(a)}{a H(a)}\right] D_{a}(a) \\
-\left[\frac{4 \pi G \rho_{m}(a)}{H^{2}(a)}-\frac{2 Q(a)}{H(a)}-a \frac{Q_{a}(a)}{H(a)}\right] \frac{D(a)}{a^{2}}=0,
\end{aligned}
$$

where $X_{a}=d X / d a$ and $X_{a a}=d^{2} X / d a^{2}$. In particular, notice that for $C_{H}=C_{\dot{H}}=0$, Eq. (18) tells us that $Q=0$ and then (24) reduces to the standard perturbation Eq. [57].
Let us now comment on the effective approach to perturbations based on Eq. (24). Use of this equation implies a treatment of matter perturbations in which the dynamical vacuum energy density is included only at the background level; that is to say, we do not consider the vacuum energy perturbations. We proceed within this approximation because it is the simplest possible way to discuss the perturbations of the entropic models under consideration, which are themselves effective models of the dark energy. We keep in mind the results from previous calculations of cosmic perturbations in models where the dark energy component was also parametrized as a power series of the Hubble function, with or without exchange of energy with the matter component (and including in some cases a possible time variation of the gravitational coupling). In these studies (see Refs. [55,56]), it is found that the full perturbative treatment of matter and dark energy perturbations in two different gauges (synchronous and conformal Newtonian) leads to consistent results. Furthermore, the confrontation of the models with the basic cosmological data produces similar results to those obtained from the effective treatment where the dynamical character of the dark energy is encoded as in Eq. (24) [37,38]. We do not exclude, however, that ambiguities can still be present in the perturbation equations and the assumptions made in their derivation. As indicated in the aforementioned references, the gauge issues can be important, and in our case the lack of a manifest covariant formulation can also play a role in this issue. For the usual scales explored in the analysis of the matter power spectrum, at sub-Hubble domains, we assume that our effective approach reflects the basic features.

Using (8), (11), and (12), Eq. (24) can be cast as follows:

$$
a^{2} D_{a a}(a)+\frac{3}{2} A a D_{a}(a)-\frac{3}{2} B D(a)=0,
$$

where

$$
\begin{aligned}
A & =\frac{1+3 C_{H}-4 \alpha}{1-\alpha}=4-3 \xi, \\
B & =\frac{\left(1+C_{H}\right)\left(1-3 C_{H}\right)+4 \alpha\left(2 C_{H}-\alpha\right)}{(1-\alpha)^{2}} \\
& =(3 \xi-2)(2-\xi) .
\end{aligned}
$$

We recall that $\xi_{\omega}=\xi\left(\omega_{m}=0\right)$ in the structure formation epoch, with $\xi$ defined in (9).

As a particular case, we consider the situation corresponding to $C_{\dot{H}}=0(\alpha=0)$. In this case $\xi=1-C_{H}$, and we immediately recover the results obtained for the $\rho_{\mathrm{DE}} \sim H^{2}$ model studied in [37]. The corresponding perturbations, Eq. (25), take the form

$$
\begin{aligned}
a^{2} D_{a a}(a) & +\frac{3}{2}\left(1+3 C_{H}\right) a D_{a}(a) \\
& -\frac{3}{2}\left(1+C_{H}\right)\left(1-3 C_{H}\right) a^{2} D(a)=0 .
\end{aligned}
$$


The general solution reads

$$
D(a)=C_{1} a^{1-3 C_{H}}+C_{2} a^{-3\left(1+C_{H}\right) / 2} .
$$

As we can see, for $\left|C_{H}\right|<1$ (the expected situation) only the $a^{1-3 C_{H}}$ mode is a growing one, provided $C_{H}<1 / 3$ (equivalent to $\xi>2 / 3$ for $C_{\dot{H}}=0$ ). However, this option for a growing mode is incompatible with having positive acceleration (or $q<0$ ) for this cosmology, as we have shown in the previous section-see Eq. (15).

As a matter of fact, this situation will replicate for the entire class of entropic models of the form Eq. (4) and not only for those with $C_{\dot{H}}=0$. Indeed, the general solution of (25) is a linear combination of the modes $a^{r_{+}}$and $a^{r_{-}}$, with

$$
r_{ \pm}=\frac{1}{2}\left(1-\frac{3}{2} A \pm \sqrt{\Delta}\right), \quad \Delta \equiv\left(1-\frac{3}{2} A\right)^{2}+6 B .
$$

We can check that for $C_{\dot{H}}=0$ these modes reduce to the ones found in the particular case (28).

In order to avoid oscillatory solutions in the general case $C_{\dot{H}} \neq 0$, we will consider only situations in which the discriminant $\Delta>0$. A growing mode solution of (25) will exist if $r_{+}>0$ and/or $r_{-}>0$. We have two possibilities: (i) if $B>0$, then $r_{+}>0$ and $r_{-}<0$ irrespective of the sign of $1-3 A / 2$, so we have one growing mode; and (ii) if $B<0$ and $1-3 A / 2>0$, we have two growing modes: $r_{+}>0$ and $r_{-}>0$. For completeness, we note that if $B<0$ and $1-3 A / 2<0$, then $r_{+}<0$ and $r_{-}<0$, and in this case we have no growing modes at all.

Let us now analyze if any of the two possibilities (i) or (ii) can be realized in practice. From the explicit form of $B$ as a function of $\xi$ in Eq. (26), we can see immediately that $B>0$ is equivalent to require $2 / 3<\xi<2$. However, this is incompatible with the acceleration condition $\xi<2 / 3$. Thus, $B>0$ is not acceptable.

Similarly, let us consider if the scenario (ii) is feasible. The condition $B<0$ implies either $\xi<2 / 3$ or $\xi>2$. Only the first case is compatible with the positive acceleration condition $\xi<2 / 3$. However, let us recall that scenario (ii) requires the additional inequality $1-3 A / 2>0$ in order to ensure the existence of growing modes. Using (26), the latter inequality can be equivalently expressed as $\xi>10 / 9$. Therefore, the intersection of positive acceleration with the existence of growing modes finally yields $\xi>2$. But this possibility is also unacceptable because from Eq. (13) we realize that this would entail $\Omega_{m 0}>2$ for entropic-force scenarios. As a result all entropic-force scenarios with values of $\Omega_{m 0}$ in a reasonable range (in particular, the favorite range $\Omega_{m 0} \simeq 0.27-0.30$ ) are incompatible with the existence of growing modes for structure formation.

The upshot of this analysis is that there are no acceptable phenomenological scenarios for the entropicforce cosmology in its original formulation [40]. The model fails both at the background level and at the perturbation level. The last feature is important for the following reason: if the failure at the background level concerning the absence of a transition point from deceleration to acceleration would be just the late-time effective behavior of a more general model where the transition would occur, not even this possibility would be allowed since the model has no growing modes for the structure formation in the late-time Universe. The core of the problem stems once more from the structure of the DE evolution law of the entropic-force model, Eq. (4), which does not have an additive constant and, therefore, has no $\Lambda \mathrm{CDM}$ limit for any value of the parameter space.

\section{AN ALTERNATIVE ENTROPIC MODEL}

In the absence of a constant term in Eq. (4), the question that we want to address now is the following: if we replace the term $C_{\dot{H}} \dot{H}(t)$ with $C_{1} H(t)$, then is it possible to provide a viable look-alike entropic-force model? Our aim is to see if one can introduce a change in the structure of the model (2) such that at least one (or maybe two) of the previous problems can be fixed. Since higher powers of $H$ (say $H^{3}$ or $H^{4}$ [58]) can have no phenomenological significance in the late Universe (as they are too small), we used just $H$. We were motivated also by the fact that vacuum models with a linear term in $H$ have also been discussed previously in the literature trying to explain the value of the cosmological constant problem within QCD [59-62].

In this case, the corresponding effective DE density is

$$
\rho_{\mathrm{DE}}(t)=\frac{3}{8 \pi G}\left[C_{1} H(t)+C_{H} H^{2}(t)\right],
$$

where here $C_{1}$ is of course a dimensionful new constant with the same dimensions as $H$. Combining Eqs. (3), (5), and (30), we find

$$
\dot{H}+\frac{3}{2}\left(1+\omega_{m}\right)\left(1-C_{H}\right) H^{2}-\frac{3}{2} C_{1} H=0 .
$$

The solution of it is

$$
H(t)=\frac{C_{1}}{\xi_{\omega}} \frac{\mathrm{e}^{3 C_{1} t / 2}}{\mathrm{e}^{3 C_{1} t / 2}-1},
$$

where $\xi_{\omega}=\xi_{1}\left(1+\omega_{m}\right)$ with $\xi_{1}=1-C_{H}$. This parameter is indicated distinctively with respect to the last section to make clear that the structure of the model is indeed different and, hence, the comparison with the data should produce in general different numerical results.

Upon a new integration it is easy to prove that the scale factor of the Universe becomes

$$
a(t)=a_{1}\left(\mathrm{e}^{3 C_{1} t / 2}-1\right)^{2 / 3 \xi_{\omega}},
$$


where $a_{1}$ is the constant of integration. Combining the above equations, we are led to a first expression for the Hubble function in terms of the scale factor:

$$
H(a)=\frac{C_{1}}{\xi_{\omega}}\left[1+\left(\frac{a}{a_{1}}\right)^{-3 \xi_{\omega} / 2}\right] .
$$

Below we focus on the matter dominated epoch, namely, $\omega_{m}=0$ and $\xi_{\omega}=\xi_{1}$. Evaluating Eq. (34) at the present time $(a \equiv 1)$, we obtain

$$
C_{1}=\frac{\xi_{1} H_{0}}{1+a_{1}^{3 \xi_{1} / 2}}
$$

Since the current value of the DE density (30) must match the measured value of the vacuum energy density $\rho_{\mathrm{DE}}\left(t_{0}\right)=\rho_{\Lambda 0}=\Omega_{\Lambda 0} \rho_{c 0}=\left(1-\Omega_{m 0}\right) 3 H_{0}^{2} / 8 \pi G$, we can determine the coefficients

$$
a_{1}=\left(\frac{\Omega_{m 0}}{\xi_{1}-\Omega_{m 0}}\right)^{2 / 3 \xi_{1}}, \quad C_{1}=H_{0}\left(\xi_{1}-\Omega_{m 0}\right) .
$$

As we can see, the two natural independent parameters of this model to be fitted to the data are $\left(\Omega_{m 0}, \xi_{1}\right)$.

Using the relations (36) in (34), we obtain the explicit form of the normalized Hubble parameter as a function of the scale factor:

$$
E(a)=\frac{H(a)}{H_{0}}=1-\frac{\Omega_{m 0}}{\xi_{1}}+\frac{\Omega_{m 0}}{\xi_{1}} a^{-3 \xi_{1} / 2} .
$$

It correctly satisfies $E(a=1)=1$ in the matter dominated epoch. ${ }^{2}$ Obviously the following condition must hold: $\xi_{1}>$ $\Omega_{m 0}\left(\right.$ or $\left.C_{H}<\Omega_{\Lambda 0}\right)$.

Finally, using the above equations, the scale factor of the Universe, normalized to unity at the present epoch, becomes

$$
a(t)=\left(\frac{\Omega_{m 0}}{\xi_{1}-\Omega_{m 0}}\right)^{2 / 3 \xi_{1}}\left[\mathrm{e}^{3 H_{0}\left(\xi_{1}-\Omega_{m 0}\right) t / 2}-1\right]^{2 / 3 \xi_{1}} .
$$

In the model under consideration, there exists a transition epoch in which the Hubble expansion changes from the decelerating to the accelerating regime $(\ddot{a}=0)$. This is in contrast to the model considered in the previous section based on the DE density (4). For the current model the inflection point can be readily calculated from the explicit form (37) setting $q=0$ in the formula (15). In contrast to the previous model, in this case it does not give a constant

\footnotetext{
${ }^{2}$ When we include later on the CMB shift parameter in the statistical analysis we need to include the radiation component in the Hubble function. We assume it is strictly conserved, i.e., $\rho_{r}=\rho_{r 0} a^{-4}$, with $\Omega_{r 0}=4.153 \times 10^{-5} h^{-2}$ [63] and $h=0.674$. We can effectively include radiation in the context of Eq. (37) by adding a term $\Omega_{r 0}\left(a^{-2}-1\right)$.
}

and, hence, it determines the following value of the scale factor and corresponding redshift:

$$
a_{I}=\frac{1}{1+z_{I}}=\left[\frac{\left(3 \xi_{1}-2\right) \Omega_{m 0}}{2\left(\xi_{1}-\Omega_{m 0}\right)}\right]^{2 / 3 \xi_{1}} .
$$

It becomes clear that the transition epoch is present only for $\xi_{1}>2 / 3$ (i.e., $C_{H}<1 / 3$ ).

While the model of the previous section was discarded both at the background and perturbation levels from pure analytical considerations, in the present case the model cannot be ruled out on the same grounds and we have to further check it by comparison with the cosmological data.

Using a joint statistical analysis, involving the latest observational data (SNIa-Union2.1 [64], BAO [65,66], and the Planck CMB shift parameter $[8,63])$, an efficient test can be implemented. Notice that the corresponding covariances can be found in Basilakos et al. [67] for the SNIa/ $\mathrm{BAO}$ data and in [63] for the Planck CMB shift parameter, respectively. We find that the overall likelihood function peaks at $\Omega_{m 0}=0.296 \pm 0.017, \xi_{1}=1.189 \pm 0.008$ with $\chi_{\min }^{2}\left(\Omega_{m 0}, \xi_{1}\right) \simeq 568.3$, resulting in a reduced value of $\chi_{\min }^{2} /$ d.o.f. $\sim 0.97$. With these numerical values we see from (36) that $C_{1}$ is of order $H_{0}$, as could be expected. At the same time we find that the transition epoch is taking place at $a_{I} \sim 0.47$ which corresponds to a redshift $z_{I} \sim 1.13$. This value is substantially higher than in the case of the concordance $\Lambda \mathrm{CDM}$ model, namely, $z_{I, \Lambda} \sim 0.70$. However, this is not the main problem; we still have to attend the analysis of matter perturbations.

\section{A. The growth factor}

In light of the new growth data (as collected by [67]) we compare the growth of matter fluctuations of the effective entropic-force model with observations. Following the procedure of $[37,68]$, to which we refer the reader for more details, we introduce the new variable

$$
y=\exp \left(3 C_{1} t / 2\right) \quad \text { with } \quad 0<y<1,
$$

and using Eqs. (3), (18), and (30), we can write

$$
\begin{aligned}
\rho_{m} & =\frac{3 C_{1}^{2} y}{\xi_{1}(y-1)^{2}} \\
Q & =\frac{3 C_{1}\left[\left(2-\xi_{1}\right) y-\xi_{1}\right]}{2 \xi_{1}(y-1)} \\
\dot{Q} & =\frac{9 C_{1}^{2}\left(\xi_{1}-1\right) y}{2 \xi_{1}(y-1)^{2}} .
\end{aligned}
$$

Inserting Eqs. (40) and (41) into Eq. (19), we arrive at the following differential equation: 


$$
\begin{gathered}
3 \xi_{1}^{2} y(y-1)^{2} D^{\prime \prime}+2 \xi_{1}(y-1)\left(5 y-3 \xi_{1}\right) D^{\prime} \\
\quad-2\left(2-\xi_{1}\right)\left(3 \xi_{1}-2 y\right) D=0,
\end{gathered}
$$

where primes indicate derivatives with respect to the new variable $y$. The latter is related with the scale factor as follows:

$$
y=1+\frac{\xi_{1}-\Omega_{m 0}}{\Omega_{m 0}} a^{3 \xi_{1} / 2} .
$$

The decaying solution of Eq. (42) can be identified easily: it reads $D_{-}(y)=(y-1)^{\left(\xi_{1}-2\right) / \xi_{1}} \sim a^{3\left(\xi_{1}-2\right) / 2}$ for $\xi_{1}<8 / 3$ (hence, $C_{H}>-5 / 3$ ). Thus, the corresponding growing mode of Eq. (42) is

$$
D_{+}(a)=C a^{3\left(\xi_{1}-2\right) / 2} \int_{0}^{a} \frac{\mathrm{d} x}{x^{3 \xi_{1} / 2} E^{2}(x)},
$$

where

$$
C=\frac{3 \Omega_{m 0}^{2}}{2 \xi_{1}}\left(\frac{\xi_{1}-\Omega_{m 0}}{\Omega_{m 0}}\right)^{2\left(3 \xi_{1}-2\right) / 3 \xi_{1}}
$$

We point out that in the limit when the linear term in the Hubble function of the DE density (30) vanishes $\left(C_{1} \rightarrow 0\right)$, the above formulas simplify dramatically. In this case the cosmological solution takes on the much simpler form:

$$
E(a) \sim a^{-3 \xi_{1} / 2}, \quad D_{+}(a) \sim a^{3 \xi_{1}-2} .
$$

The resulting DE model is of course coincident with the situation $C_{\dot{H}}=0$ studied for the model of Sec. III, which was problematic because the existence of growing modes is incompatible with having accelerated expansion; see Eq. (28).

Finally, let us consider the evolution of the growth rate of clustering, defined as $f(a)=d \ln D_{+} / d \ln a$. It can be computed from the growing mode solution (44). We find

$$
f(a)=\frac{3\left(\xi_{1}-2\right)}{2}+\frac{C}{a^{2} E^{2}(a) D_{+}(a)} .
$$

In Fig. 1, we plot the growth data as collected by Basilakos et al. (see [67] and references therein) with the estimated growth rate function, $f(z) \sigma_{8}(z)$. The solid line in the figure corresponds to the aforementioned best fit values for SNIa/BAO data and the Planck CMB shift parameter, i.e., for $\left(\Omega_{m 0}, \xi_{1}\right)=(0.296,1.188)$. We have included also the prediction of the concordance $\Lambda \mathrm{CDM}$ model - see the dashed line. Note that the theoretical $\sigma_{8}(z)$ is given by $\sigma_{8}(z)=\sigma_{8} D(z)$, where $D(z)=D_{+}(z) / D_{+}(z=0)$ is the growth factor scaled to unity at the present time, and $\sigma_{8}$ is the rms mass fluctuation on $R_{8}=8 h^{-1} \mathrm{Mpc}$ scales at redshift $z=0$. We use for it the Planck parametrization value: $\sigma_{8}=0.818$ [69].

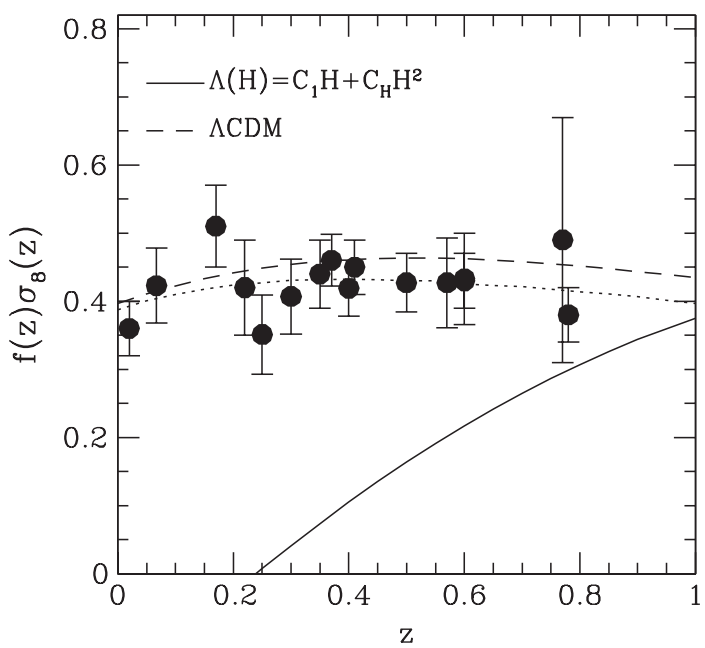

FIG. 1. Comparison of the observed (solid points) and theoretical evolution of the growth rate $f(z) \sigma_{8}(z)$. The solid line corresponds to the alternate entropic-force DE model (30) for the best fit values $\left(\Omega_{m 0}, \xi_{1}\right)=(0.296,1.189)$ discussed in Sec. IV. The dotted line shows the predicted growth rate in the case of $\left(\Omega_{m 0}, \xi_{1}\right)=(0.73,1.01)$. For comparison we also plot the $\Lambda$ CDM (dashed line). We use $\sigma_{8}=0.818$ [69], while for the $\Lambda$ CDM case we set $\Omega_{m 0}=0.272$ [67].

From the comparison it becomes clear that the present growth data strongly disfavor the alternate entropic-force DE model (30). The lack of structure formation near our time is quite evident as compared to the concordance $\Lambda \mathrm{CDM}$ model. If we, however, enforce that the model optimally fits only the data on the growth rate, we find $\xi_{1} \simeq 1.01$ and $\Omega_{m 0} \simeq 0.73$ (see the dotted line in Fig. 1). Obviously, the obtained value of $\Omega_{m 0}$ is far beyond the acceptable physical range of values and, thus, it must be rejected.

Let us also mention that the DE model (30) with $\xi_{1} \rightarrow 1$ (i.e., $C_{H} \rightarrow 0$ ) reduces to that of Borges et al. $[54,62]$ in which the DE term is directly proportional to the Hubble parameter, $\rho_{\mathrm{DE}}(a) \propto H(a)$. In this framework, we repeat our joint statistical analysis by imposing $\xi_{1}=1$ into Eq. (37), and we find that the overall fit provides $\Omega_{m 0}=$ $0.30 \pm 0.01$, but with a poor quality: $\chi_{\min }^{2}\left(\Omega_{m 0}\right) /$ d.o.f. $\simeq 2.7$. The main problem here is related with the fact that the $\rho_{\mathrm{DE}}(a) \propto H(a)$ model cannot accommodate the $\mathrm{CMB}$ data. Indeed, using the Planck CMB shift parameter alone, we find that the corresponding likelihood function peaks at the totally unrealistic value $\Omega_{m 0} \simeq 0.97$, which is $\sim 3.2$ times larger than that provided by the SNIa + BAO solution $\Omega_{m 0} \simeq 0.30$.

Finally, the following observation is in order. The DE models in which the energy density is strongly dependent on odd powers of the Hubble rate are not favored from the theoretical point of view [34-36], as it is impossible to fit them into the covariant form of the effective action of QFT in curved spacetime. If they contain a combination of even and odd powers of $H$, one can treat the odd powers 
phenomenologically as representing, e.g., bulk viscosity effects. However, only the even powers can have a fundamental origin. In this sense, DE models based on pure linear terms in $H$ are not well motivated, and in fact we find that they fit the main cosmological data poorly. We have seen that after including the $H^{2}$ term in it, as in Eq. (4), the new DE density behaves better at the background level since it provides an acceptable fit value around $\Omega_{m 0} \sim 0.3$. Notwithstanding, it does not pass the stringent test of the structure formation data.

Overall, the above analysis points out that even if we replace the term $C_{\dot{H}} \dot{H}(t)$ in Eq. (4) with $C_{1} H(t)$, the modified DE model, namely, Eq. (30), is unable to fit simultaneously the observational data on Hubble expansion and structure formation.

\section{CONCLUSIONS}

In this work we have considered the entropic-force model [39] and reanalyzed the implications as a possible dark energy candidate in cosmology within the context of [40]. We have found that in its original formulation, the effective dark energy implied by such a model is not viable either at the background level or at the cosmic perturbation level. Specifically, the analysis of the deceleration parameter immediately detects a fundamental problem, to wit: the expansion of the Universe always accelerates or always decelerates. While this is of course a severe problem, it might still be cured if such behavior were only the late-time behavior of a more complete model where the transition from deceleration to acceleration would be present. However, a detailed analysis of the cosmic perturbations furnishes also a negative result, and this may give the final blow to the model: it turns out that the existence of growing modes for structure formation is incompatible with the accelerated expansion.

In an attempt to rescue some form of the entropic-force DE model, we have also addressed a modification of its energy density by replacing the time derivative term $\dot{H}$ with the linear term $H$, while keeping the $H^{2}$ one. The modified model is better behaved at the background level, because there is a transition point from deceleration to acceleration and, moreover, the best fit value of $\Omega_{m 0}$ is near 0.3 . However, the modified model is once more unable to fit the most recent growth data on structure formation. Let us mention that if we would also suppress the $H^{2}$ component (i.e., if the DE density would be reduced to the linear term in the Hubble function), then the best fit value of $\Omega_{m 0}$ has poor quality in the allowed region and runs completely out of range (near 1) if we single out the CMB data.

In all these cases, the absence of an additive term in the structure of the DE density lies at the root of the main difficulties with these models. This constant is essential for the transition from deceleration into acceleration. It is also essential to provide a late-time linear growth rate that can describe the structure formation data in a way that is comparable to the $\Lambda \mathrm{CDM}$ model. If the structure of the entropic-force models is corrected with an additive term, i.e., if the DE density becomes an "affine function" of the Hubble terms, $\rho_{\mathrm{DE}}=c_{0}+c_{1} H+c_{\dot{H}} \dot{H}+c_{2} H^{2}$, then these models have a well-defined $\Lambda \mathrm{CDM}$ limit when the coefficients of the $H, \dot{H}$, and $H^{2}$ terms approach zero. These modified models can be phenomenologically compatible with the cosmological data $[48,70]$.

Let us note that we have focused on the study of the entropic-force models of the $\Lambda$ type, i.e., such that the DE density (4) has the equation of state $\omega_{\mathrm{DE}}=-1$, as in Ref. [48]. One may consider other possible implementations [49,50], but to get a viable one it is unavoidable to introduce in all cases an additive term. Unfortunately this cannot be done in a natural way in the entropic-force formulation.

Such situation is in stark contradistinction to the running vacuum models based on the renormalization group in semiclassical gravity in curved spacetime [10]. The latter types of models have existed in the literature since long ago - see [34-36] and references therein-and have been successfully tested $[37,38,55,56]$. They naturally incorporate the additive term as an integration constant, namely, in the affine form mentioned above, but only the even powers of $H$ (and the powers of $\dot{H}$ ) are allowed, as expected from the covariant QFT formulation in a curved background.

The entropic-force models that have became popular lately are actually a particular case of the running vacuum models, as clearly discussed in [48]. This particular case, however, proves to be phenomenologically inviable at the background level and/or at the perturbation level, as we have clearly demonstrated here. Even if other formulations of the entropic models are possible, the main result, namely, that simple combinations of pure Hubble terms $H, \dot{H}, H^{2}$ are not sufficient for a complete description of the cosmological data, stays in force.

\section{ACKNOWLEDGMENTS}

We thank T. Padmanabhan for useful comments. S. B. acknowledges support by the Research Center for Astronomy of the Academy of Athens in the context of the program "Tracing the Cosmic Acceleration." J. S. has been supported in part by FPA2010-20807 (MICINN), Consolider Grant No. CSD2007-00042 (CPAN), and by DIUE/CUR Generalitat de Catalunya under project 2009SGR502. 
[1] D. N. Spergel et al., Astrophys. J. Suppl. Ser. 170, 377 (2007).

[2] T. M. Davis et al., Astrophys. J. 666, 716 (2007).

[3] M. Kowalski et al., Astrophys. J. 686, 749 (2008).

[4] M. Hicken, W. M. Wood-Vasey, S. Blondin, P. Challis, S. Jha, P. L. Kelly, A. Rest, and R. P. Kirshner, Astroplys. J. 700, 1097 (2009).

[5] E. Komatsu et al., Astrophys. J. Suppl. Ser. 180, 330 (2009); G. Hinshaw et al., Astrophys. J. Suppl. Ser. 180, 225 (2009); E. Komatsu et al., Astrophys. J. Suppl. Ser. 192, 18 (2011).

[6] J. A. S. Lima and J. S. Alcaniz, Mon. Not. R. Astron. Soc. 317, 893 (2000); J. F. Jesus and J. V. Cunha, Astrophys. J. Lett. 690, L85 (2009).

[7] S. Basilakos and M. Plionis, Astrophys. J. Lett. 714, L185 (2010).

[8] P. A. R. Ade et al. (Planck Collaboration), arXiv:1303.5076 [Astron. Astrophys. (to be published)].

[9] S. Weinberg, Rev. Mod. Phys. 61, 1 (1989); V. Sahni and A. Starobinsky, Int. J. Mod. Phys. D 09, 373 (2000); T. Padmanabhan, Phys. Rep. 380, 235 (2003).

[10] J. Solà, J. Phys. Conf. Ser. 453, 012015 (2013); arXiv:1402.7049.

[11] A. D. Dolgov, in The Very Early Universe, edited by G. Gibbons, S. W. Hawking, and S. T. Tiklos (Cambridge University Press, Cambridge, England, 1982); Y. Fujii, Phys. Rev. D 26, 2580 (1982).

[12] L. Abbott, Phys. Lett. 150B, 427 (1985); S. M. Barr, Phys. Rev. D 36, 1691 (1987); L. H. Ford, Phys. Rev. D 35, 2339 (1987); E. T. Tomboulis, Nucl. Phys. B329, 410 (1990).

[13] R. D. Peccei, J. Solà, and C. Wetterich, Phys. Lett. B 195, 183 (1987); J. Solà, Phys. Lett. B 228, 317 (1989); Int. J. Mod. Phys. A 05, 4225 (1990).

[14] C. Wetterich, Nucl. Phys. B302, 668 (1988); B. Ratra and P. J. E. Peebles, Phys. Rev. D 37, 3406 (1988); C. Wetterich, Astron. Astrophys. 301, 321 (1995); R. R. Caldwell, R. Dave, and P. J. Steinhardt, Phys. Rev. Lett. 80, 1582 (1998).

[15] P. J. Peebles and B. Ratra, Rev. Mod. Phys. 75, 559 (2003).

[16] S. Capozziello, Int. J. Mod. Phys. D 11, 483 (2002).

[17] S. Capozziello and M. Francaviglia, Gen. Relativ. Gravit. 40, 357 (2008).

[18] S. Capozziello and M. De Laurentis, Phys. Rep. 509, 167 (2011).

[19] S. Nojiri and S. D. Odintsov, Phys. Rep. 505, 59 (2011).

[20] M. Ozer and O. Taha, Nucl. Phys. B287, 776 (1987).

[21] W. Chen and Y.-S. Wu, Phys. Rev. D 41, 695 (1990); J. C. Carvalho, J. A. S. Lima, and I. Waga, Phys. Rev. D 46, 2404 (1992); J. A. S. Lima and J. M. F. Maia, Phys. Rev. D 49, 5597 (1994); J. A. S. Lima, Phys. Rev. D 54, 2571 (1996); A. I. Arbab and A. M. M. Abdel-Rahman, Phys. Rev. D 50, 7725 (1994); J. M. Overduin and F. I. Cooperstock, Phys. Rev. D 58, 043506 (1998).

[22] P. Brax and J. Martin, Phys. Lett. B 468, 40 (1999).

[23] A. Kamenshchik, U. Moschella, and V. Pasquier, Phys. Lett. B 511, 265 (2001).

[24] A. Feinstein, Phys. Rev. D 66, 063511 (2002).

[25] R. R. Caldwell, Phys. Lett. B 545, 23 (2002).

[26] M. C. Bento, O. Bertolami, and A. A. Sen, Phys. Rev. D 70, 083519 (2004).
[27] L. P. Chimento and A. Feinstein, Mod. Phys. Lett. A 19, 761 (2004).

[28] E. V. Linder, Rep. Prog. Phys. 71, 056901 (2008).

[29] J. A. S. Lima, F. E. Silva, and R. C. Santos, Classical Quantum Gravity 25, 205006 (2008).

[30] A. W. Brookfield, C. van de Bruck, D. F. Mota, and D. Tocchini-Valentini, Phys. Rev. Lett. 96, 061301 (2006).

[31] C. G. Boehmer and T. Harko, Eur. Phys. J. C 50, 423 (2007).

[32] A. A. Starobinsky, JETP Lett. 86, 157 (2007).

[33] E. J. Copeland, M. Sami, and S. Tsujikawa, Int. J. Mod. Phys. D 15, 1753 (2006); R. R. Caldwell and M. Kamionkowski, Annu. Rev. Nucl. Part. Sci. 59, 397 (2009); I. Sawicki and W. Hu, Phys. Rev. D 75, 127502 (2007); L. Amendola and S. Tsujikawa, Dark Energy Theory and Observations (Cambridge University Press, Cambridge, UK, 2010); S. Capozziello and V. Faraoni, Beyond Einstein Gravity: A Survey of Gravitational Theories for Cosmology and Astrophysics, Fundamental Theories of Physics Vol. 170 (Springer, Heidelberg, 2010).

[34] I. L. Shapiro and J. Solà, J. High Energy Phys. 02 (2002) 006; Phys. Lett. B 475, 236 (2000); Nucl. Phys. B, Proc. Suppl. 127, 71 (2004); arXiv:astro-ph/0401015.

[35] I. L. Shapiro and J. Solà, Phys. Lett. B 682, 105 (2009); See also the extended discussion in , arXiv:0808.0315.

[36] J. Solà, J. Phys. A 41, 164066 (2008).

[37] S. Basilakos, M. Plionis, and J. Solà, Phys. Rev. D 80, 083511 (2009).

[38] J. Grande, J. Solà, S. Basilakos, and M. Plionis, J. Cosmol. Astropart. Phys. 08 (2011) 007.

[39] E. P. Verlinde, J. High Energy Phys. 04 (2011) 029.

[40] D. A. Easson, P. H. Frampton, and G. F. Smoot, Phys. Lett. B 696, 273 (2011).

[41] D. A. Easson, P. H. Frampton, and G. F. Smoot, Int. J. Mod. Phys. A 27, 1250066 (2012).

[42] T. Jacobson, Phys. Rev. Lett. 75, 1260 (1995).

[43] T. Padmanabhan, Rep. Prog. Phys. 73, 046901 (2010); Phys. Rep. 406, 49 (2005).

[44] M. Visser, arXiv:1108.5240.

[45] M. Li and Y. Wang, Phys. Lett. B 687, 243 (2010).

[46] R. Casadio and A. Gruppuso, Phys. Rev. D 84, 023503 (2011); T. S. Koivisto, D. F. Mota, and M. Zumalacarregui, J. Cosmol. Astropart. Phys. 02 (2011) 027.

[47] Y.-F. Cai, J. Liu, and H. Li, Phys. Lett. B 690, 213 (2010); H. Wei, Phys. Lett. B 692, 167 (2010); Y. S. Myung, Astrophys. Space Sci. 335, 553 (2011); Y.-F. Cai and E. N. Saridakis, Phys. Lett. B 697, 280 (2011).

[48] S. Basilakos, D. Polarski, and J. Solà, Phys. Rev. D 86, 043010 (2012).

[49] N. Komatsu and S. Kimura, Phys. Rev. D 88, 083534 (2013); 87043531 (2013).

[50] N. Komatsu and S. Kimura, arXiv:1402.3755 [Phys. Rev. D (to be published)].

[51] S. W. Hawking and G. T. Horowitz, Classical Quantum Gravity 13, 1487 (1996).

[52] J. Grande, A. Pelinson, and J. Solà, Phys. Rev. D 79, 043006 (2009).

[53] R. C. Arcuri and I. Waga, Phys. Rev. D 50, 2928 (1994).

[54] H. A. Borges, S. Carneiro, J. C. Fabris, and C. Pigozzo, Phys. Rev. D 77, 043513 (2008). 
[55] J. Grande, J. Solà, J. C. Fabris, and I. L. Shapiro, Classical Quantum Gravity 27, 105004 (2010).

[56] J. C. Fabris, I. L. Shapiro, and J. Solà, J. Cosmol. Astropart. Phys. 02 (2007) 016; A. M. Velasquez-Toribio, Int. J. Mod. Phys. D 21, 1250026 (2012).

[57] P. J. E. Peebles, Principles of Physical Cosmology (Princeton Univ. Press, Princeton, New Jersey, 1993); S. Dodelson, Modern Cosmology (Academic Press, New York, 2003).

[58] J. A. S. Lima, S. Basilakos, and J. Solà, Mon. Not. R. Astron. Soc. 431, 923 (2013); E. L. D. Perico, J. A. S. Lima, S. Basilakos, and J. Solà, Phys. Rev. D 88, 063531 (2013); S. Basilakos, J. A. S. Lima, and J. Solà, Int. J. Mod. Phys. D 22, 1342008 (2013).

[59] R. Schutzhold, Phys. Rev. Lett. 89, 081302 (2002).

[60] F. R. Klinkhamer and G. E. Volovik, Phys. Rev. D 79, 063527 (2009).

[61] E. C. Thomas, F. R. Urban, and A. R. Zhitnitsky, J. High Energy Phys. 08 (2009) 043; N. Ohta, Phys. Lett. B 695, 41 (2011).
[62] S. Carneiro, M. A. Dantas, C. Pigozzo, and J. S. Alcaniz, Phys. Rev. D 77, 083504 (2008).

[63] D. L. Shaefer and D. Huterer, Phys. Rev. D 89, 063510 (2014).

[64] N. Suzuki, D. Rubin, C. Lidman, G. Aldering, R. Amanullah, K. Barbary, L. F. Barrientos, J. Botyanszki et al., Astrophys. J. 746, 85 (2012).

[65] C. Blake, E. Kazin, F. Beutler, T. Davis, D. Parkinson, S. Brough, M. Colless, C. Contreras et al., Mon. Not. R. Astron. Soc. 418, 1707 (2011).

[66] W. J. Percival, Mon. Not. R. Astron. Soc. 401, 2148 (2010).

[67] S. Basilakos, S. Nesseris, and L. Perivolaropoulos, Phys. Rev. D 87, 123529 (2013).

[68] S. Basilakos, Mon. Not. R. Astron. Soc. 395, 2347 (2009).

[69] D. Spergel, R. Flauger, and R. Hlozek, arXiv:1312.3313.

[70] S. Basilakos, A. Gómez, and J. Solà (to be published). 